\title{
Relationship between past myocardial infarction, periodontal disease and Porphyromonas gingivalis serum antibodies: A case-control study
}

\author{
Radosław P. Łysek ${ }^{1}$, Krystyna Szafraniec ${ }^{1}$, Maciej Polak ${ }^{1}$, Piotr Jankowski ${ }^{2}$, \\ Agnieszka Micek ${ }^{1}$, Renata Wolfshaut-Wolak ${ }^{1}$, Danuta Czarnecka ${ }^{2}$, \\ Jan Potempa ${ }^{3,4}$, Andrzej Pająk ${ }^{1}$ \\ ${ }^{1}$ Department of Epidemiology and Population Studies, Institute of Public Health, \\ Jagiellonian University Medical College, Krakow, Poland \\ ${ }^{2} 1^{\text {st }}$ Department of Cardiology, Electrocardiology and Hypertension, \\ Jagiellonian University Medical College, Krakow, Poland \\ ${ }^{3}$ Department of Microbiology, Faculty of Biochemistry, Biophysics and Biotechnology, \\ Jagiellonian University, Krakow, Poland \\ ${ }^{4}$ Department of Oral Immunology and Infectious Diseases, \\ University of Louisville School of Dentistry, Louisville, KY, USA
}

\begin{abstract}
Background: The relationship between chronic periodontitis (CP) and increased risk for cardiovascular disease (CVD) is known but quantitative assessments and mechanisms are not fully understood. The aim of this study was to assess the relationship between past myocardial infarction (MI) and the severity of $C P$, and the level of serum antibody titer against Porphyromonas gingivalis gingipains.

Methods: The study sample consisted of 97 patients after MI and 113 high risk controls with no history of coronary heart disease (CHD) matched with age, sex and place of residence (urban vs. rural). Data on the history of CHD and presence of risk factors were collected. Periodontal status was assessed using the Community Periodontal Index (CPI), clinical attachment loss (CAL), bleeding on probing (BOP) and pocket depth.

Results: After adjustment for potential confounders patients with $B O P=20-50 \%$ and $B O P>50 \%$ had more than four times higher odds of past $M I(O R=4.56 ; 95 \%$ CI 2.03-10.27). Patients with CPI code $=4$ had a three times higher odds of past $M I(O R=3.18,95 \%$ CI 1.01-10.06). CAL $\geq 6$ was related to higher odds of past $M I(O R=1.28,95 \%$ CI 1.11-1.49). Patients with moderate antibody titer levels had an almost 3 times higher odds of past MI (OR $=2.82,95 \%$ CI 1.02-7.84).

Conclusions: There was an association between CP and past MI, which was independent of classical $C V D$ risk factors and confirmed by an association between past $M I$ and immunological reaction against P. gingivalis gingipains. (Cardiol J 2018; 25, 3: 386-392)
\end{abstract}

Key words: periodontal disease, myocardial infarction, Porphyromonas gingivalis gingipains, cardiovascular disease risk factors

Address for correspondence: Radosław P. Łysek, MD, PhD, Department of Epidemiology and Population Studies, Institute of Public Health, Jagiellonian University Medical College, ul. Grzegórzecka 20, 31-531 Kraków, Poland, tel: +48 124332839 , e-mail: radoslaw.lysek@gmail.com

Received: 14.06.2016 Accepted: 14.08.2016 


\section{Introduction}

An increasing number of studies have shown a relationship between chronic periodontitis $(\mathrm{CP})$ and increased risk of cardiovascular disease (CVD) even after controlling for possible confounders $[1,2]$. CP has been postulated to be a CVD risk factor, which is independent of well-known risk factors, such as smoking, hypertension, obesity, diabetes and hypercholesterolemia [3, 4]. CP is an inflammatory disease of tooth supporting tissues and is commonly prevalent among the middle-aged and the elderly in the general population. The prevalence of $\mathrm{CP}$ in the general European population is estimated to be over $50 \%$, and the percentage of patients with severe forms of CP is over 20\% [5]. Its prevalence increases with age. According to a recent nationwide Polish study, almost $20 \%$ of the population from large Polish cities aged 35-44 years and almost $32 \%$ aged $65-74$ years had severe CP [6].

The most pathogenic and etiologic bacteria for CP are: Porphyromonas gingivalis, Bacteroides forsythus, Treponema denticola, Eikenella corrodens, Campylobacter rectus, Prevotella intermedia, and Fusobacterium nucleatum [7]. P. gingivalis is the most frequent bacteria and is identified in $85 \%$ of sites of CP [8]. P. gingivalis has a number of potential virulence factors, such as cysteine proteinases — gingipains (GPs), lipopolysaccharide, capsules, and fimbriae [9]. GPs contribute to $85 \%$ of the total proteolytic activity of $P$. gingivalis $[10]$ and are crucial in inducing $\mathrm{CP}$ since they have multidirectional adverse impacts on the immune system. The patomechanism of the relation between $\mathrm{CP}$ and coronary heart disease (CHD) is still unclear, but in general two mechanisms have been postulated. The first is the general inflammatory host response in CP, which is supported by the findings of elevated inflammatory markers (C-reactive protein) in $\mathrm{CP}$ patients and their decrease after successful treatment of periodontitis [11]. Furthermore, it has been found that $P$. gingivalis may increase overall inflammatory response through its virulence factor, especially GPs $[12,13]$. The second postulated mechanism involves direct invasion of arteries and endothelial cells by periodontal pathogens, especially $P$. gingivalis. The latter is supported by findings that endothelial dysfunction, which plays a key role in the development of arteriosclerotic plaques, was associated with CP [14]. Furthermore, antibodies against $P$. gingivalis have been found in human arteriosclerotic plaques [15] and experimentally induced $P$. gingivalis bacteremia accelerated formation of arteriosclerotic plaques in pigs [16]. In addition, defects in endothelial cells and arteriosclerotic plaques that appear as the effect of CP may be a background for myocardial infarction (MI) [15].

In most studies showing an association between $\mathrm{CP}$ and $\mathrm{CHD}$, periodontal status was based on clinical examination or self-reporting. Such methods do not allow for the assessment of systematic immunological response to $\mathrm{CP}$. The level of antibodies against periodontopathogenic bacteria, especially their major virulence factors as a better indicator of the $\mathrm{CP}$ [17] could be a more accurate measure of the link between $\mathrm{CP}$ and systemic diseases. Therefore the aim of this study was to assess the association between past MI and the clinical parameters of $\mathrm{CP}$ in the context of the level of serum antibody titer against $P$. gingivalis gingipains.

\section{Methods}

\section{Study design}

A frequency-matched case-control study was performed to identify factors which are associated with past MI that are related to the periodontium health status. The potential characteristics explored were: pocket depth (PD), clinical attachment loss (CAL), bleeding on probing (BOP), community periodontal index (CPI), and the titer of antibody (AT) directed against gingipains. The study group consisted of 220 patients, including 97 cases and 113 controls.

For a type I error of $5 \%$ and power of $80 \%, 98$ cases were sufficient to detect an odds ratio $>2.2$ for factors with a prevalence of $35 \%$ in the general population [18].

\section{Studied groups}

Patients after MI were recruited from five cardiology departments, serving the city of Krakow and the surrounding rural area. Patients aged $\geq 18$ years and $\leq 80$ years, with definite clinical diagnosis of MI, were identified from medical records, excluding those who died during their in-hospital stay and were asked to undergo dental examination.

Controls were persons with no diagnosis of $\mathrm{MI}$ or $\mathrm{CHD}$, who were selected randomly from three general practices: two in Krakow and one outside of Krakow. Inclusion criteria were: high CVD risk identified by attempted treatment for hypertension, hypercholesterolemia or diabetes mellitus. Information on treatment was collected from medical records using a standardized data collection form. Then, controls were matched ac- 
cording to their age (5-year groups), sex and place of residence (rural vs. urban) and were asked to undergo dental examination. However, of the 113 controls, $91(80.5 \%)$ were comprised of men and $22(19.5 \%)$ women, i.e. 5 women less than in the previous MI group. Those 5 women were excluded because they had less than 6 teeth.

In both groups, the exclusion criteria were: use of antibiotics during the 2 weeks prior to the examination, and immunosuppressive therapy or chemotherapy.

\section{Data collection}

Data on demographic characteristics, personal history of CHD, smoking status, blood pressure, fasting glucose, plasma lipids and prescribed medications were obtained using a standardized data collection form. Patient height and weight were measured in a standing position without shoes and heavy outer garments using standard scales with a vertical ruler. Blood pressure was measured twice, on the right arm in a sitting position after at least $5 \mathrm{~min}$ of rest. For plasma lipid and glucose measurements, a fasting venous blood sample was taken between 7:30 AM and 8:30 AM. Biochemical analyses were carried out no later than $4 \mathrm{~h}$ after blood collection. All analyses were performed at one central laboratory.

\section{Dental examination}

Periodontal condition was assessed in participants with at least 6 teeth. Dental clinical examination was carried out according to World Health Organization recommendations [19]. Participants were examined by one qualified dentist. Standard intraoral examination was performed starting from the first to the fourth quadrant. Pocket depth was measured on 6 sites around each tooth using a PCPUNC15-type periodontal Hu-Friedy probe. BOP was evaluated according to Ainamo and Bay [20]. For each patient, the percentage of teeth with bleeding after gentle probing was calculated. Results were interpreted as follows: $\mathrm{BOP}>50 \%$ severe, advanced $\mathrm{CP}, \mathrm{BOP}=20-50 \%$ moderate $\mathrm{CP}$, $\mathrm{BOP}=19-10 \%$ mild $\mathrm{CD}, \mathrm{BOP}<10 \%$ no $\mathrm{CP} . \mathrm{CAL}$ was defined as the distance between the pocket bottom and the cement-enamel junction. The CPI was used to record periodontal status [21]. CPI ranged from 0 to 4 , representing healthy (CPI 0 ), bleeding on probing (CPI 1), calculus or conditions for dental plaque retention (CPI 2), $4-5 \mathrm{~mm}$ pocket depth (CPI 3) and $\geq 6 \mathrm{~mm}$ pocket depth (CPI 4). Participants were classed into CPI groups according to the worst measurement in any quadrant.
Serum samples for antibody determination

Serum samples for antibody determination were stored at $-80^{\circ} \mathrm{C}$ immediately after centrifugation. Immunoglobulin $\mathrm{G}$ antibody titers against $P$. gingivalis GPs were determined using the enzyme-linked immunosorbent assay (ELISA), in all samples using the method described by Zdzalik et al. [22]. Antibody titers are given in ELISA units.

\section{Definition of CVD risk factors}

Participants were considered smokers, if they declared they had smoked at least one cigarette during 1 month prior to the interview or had $\geq 10 \mathrm{ppm}$ carbon monoxide in their exhaled air. The concentration of carbon monoxide in exhaled air was measured using Smokerlyzer Micro+ (Bedfont Scientific Ltd, England). Hypertension was defined as blood pressure $\geq 140 / 90 \mathrm{mmHg}$ or antihypertensive drugs taken by a patient. Hypercholesterolemia was defined as total cholesterol $\geq 5.0 \mathrm{mmol} / \mathrm{L}$ or low density lipoprotein $\geq 3.0 \mathrm{mmol} / \mathrm{L}$ or lipid lowering drugs taken by a patient. Diabetes mellitus was defined as fasting glucose $\geq 7 \mathrm{mmol} / \mathrm{L}$ or self-reported history. Obesity was defined as body mass index $\geq 30 \mathrm{~kg} / \mathrm{m}^{2}$.

\section{Statistical analysis}

All analyses were performed together for males and females. Cases and controls were compared for demographic characteristics, health status variables and oral health indices using t-Student, Mann-Whitney or $\chi^{2}$ tests as appropriate. Unconditional logistic regression was used to assess the relation between periodontal indices, level of antibody titer and past MI. Three models were examined: a univariate model (model A), and two multivariate models in which the matching variables were forced into model. Model B was adjusted for age, gender and education. The final model (model C) included additional covariates: smoking, diabetes, hypertension, hypercholesterolemia, and body mass index. Antibody titer was included in the logistic regression as a categorical variable. Based on a frequency distribution of all subjects, antibody titer values were divided into three categories and then compared between cases and controls. The reference category included the lowest 10 percent of values (AT < 900), titers above 900 to the median value $(=8,100)$ formed the second category, values above the median were included in a third category.

Sensitivity analysis that restricted cases and controls to 1:1 matching was also performed. The frequency-matched case-control analysis included 92 pairs of observations. Five cases were excluded 
Table 1. General descriptive statistics and cardiovascular disease risk factors in the study groups.

\begin{tabular}{lccc}
\hline & MI $(\mathbf{n}=\mathbf{9 7})$ & Control group $(\mathbf{n}=\mathbf{1 1 3})$ & $\mathbf{P}$ \\
\hline Age $[$ years] & $60.5 \pm 8.7$ & $60.4 \pm 8.7$ & $0.94^{\mathrm{C}}$ \\
Men & $72.2 \%$ & $80.5 \%$ & $0.15^{\mathrm{A}}$ \\
Years of education [years] ${ }^{* *}$ & $12.0(10.0-15.0)$ & $13.0(11.0-17.0)$ & $\mathbf{0 . 0 0 5 ^ { \mathrm { B } }}$ \\
Body mass index $\left[\mathrm{kg} / \mathrm{m}^{2}\right]^{*}$ & $29.0 \pm 4.7$ & $28.6 \pm 4.7$ & 0.61 \\
Arterial hypertension & $80.4 \%$ & $73.0 \%$ & 0.21 \\
Diabetes & $23.4 \%$ & $24.3 \%$ & 0.88 \\
Hyperlipidemia & $71.3 \%$ & $79.7 \%$ & 0.16 \\
Obesity & $36.1 \%$ & $31.8 \%$ & 0.52 \\
Smoking & $17.7 \%$ & $17.9 \%$ & 0.98 \\
\hline
\end{tabular}

${ }^{*}$ Mean \pm standard deviation; ** Median (upper-lower quartile); ${ }^{A} \chi^{2}$ test; ${ }^{B}$ Mann-Whitney $\mathrm{U}$ test; ${ }^{\mathrm{C}}$ Student $\mathrm{T}$ test; $\mathrm{MI}$ - myocardial infarction; $\mathrm{n}$ - number of participants

due to lack of appropriately matched controls within the gender.

Results are presented as odds ratios (OR) with 95\% confidence intervals (95\% CI). Analyses were performed using SPSS Software (IBM, Armonk, NY, USA). Statistical significance was accepted at the level of $\alpha=0.05$.

\section{Ethics}

All participants were informed about the study protocol and gave their informed consent. The study was approved by the Bioethics Committee of the Jagiellonian University.

\section{Results}

There were no significant differences in mean age and distribution by sex between the studied groups. Patients after MI were slightly less educated than controls. However, the prevalence of CVD risk factors, i.e. smoking, hypertension, hyperlipidemia, obesity, and diabetes, were similar (Table 1).

Patients after MI had a lower mean number of teeth (15 vs. 18). On average, they also had their teeth affected by periodontal disease more frequently than the controls. Cases were more likely than the controls to have some periodontal pocketing of $6 \mathrm{~mm}$ or more ( $29.9 \%$ vs. $17.7 \%$, respectively) and had a significantly higher median value of CAL (7 mm compared to $6 \mathrm{~mm}$ for controls, $\mathrm{p}<0.001$ ). They were also more likely to have a higher percentage of bleeding teeth and to have some teeth with calculus deposits or conditions for dental plaque retention. Cases had a higher percentage of participants with CPI stage 4 and BOP $>50 \%$. However, differences in antibody titer against $P$. gingivalis gingipains between the cases and the control group were not observed when compared as a quantitative variable or a categorical variable (Table 2 ).

Table 3 shows clear and consistent ORs of past MI for various periodontal health indicators.

After adjustment for age, sex, years of education, smoking, hypertension, hypercholesterolemia, body mass index, diabetes mellitus, and number of teeth, those patients with BOP $20-50 \%$ and $\mathrm{BOP} \geq 50 \%$ had a more than 4 times higher odds of past MI. Patients with CPI 4 code had a 3 more times higher odds of past $\mathrm{MI}$ and those with CAL $\geq 6 \mathrm{~mm}$ had a 1.28 odds of past MI. Association between $\mathrm{PD} \geq 6 \mathrm{~mm}$ and past MI was attenuated after adjustment for possible confounders. A clear relationship between antibody titer and the odds of past MI was not apparent in the univariate model. When some potential confounding factors were entered into regression models, those patients from antibody titer group 2,700-8,100 had an almost 3 times higher odds of past MI.

In a separate model (not shown in the tables), the sample was limited to 92 cases and their 92 matched controls. The relations between past MI, $\mathrm{CP}$ and antibody titer in the unconditional and conditional models were similar.

\section{Discussion}

These results indicate that impaired periodontal health is associated with prevalence of past MI which was confirmed also by increased antibody titer against $P$. gingivalis gingipains in patients after MI. The relation was independent of classic CVD risk factors. 
Table 2. Descriptive statistics for oral and periodontal health in the study groups.

\begin{tabular}{|c|c|c|c|}
\hline & $\mathrm{MI}(\mathbf{n}=97)$ & Control group $(n=113)$ & $\mathbf{P}$ \\
\hline Number of teeth & $15 \pm 6$ & $18 \pm 7$ & $0.0006^{A}$ \\
\hline Pocket depth [mm] & $5(4-6)$ & $5(4-5)$ & 0.12 \\
\hline Pocket depth $\geq 6 \mathrm{~mm}$ & $29.9 \%$ & $17.7 \%$ & 0.04 \\
\hline $\mathrm{CAL}[\mathrm{mm}]$ & $7(6-9)$ & $6(5-7)$ & $<0.0001$ \\
\hline BOP less than $20 \%$ teeth & $29.47 \%$ & $69.03 \%$ & $<0.001$ \\
\hline BOP $=20-50 \%$ teeth & $35.79 \%$ & $15.93 \%$ & \\
\hline BOP above $50 \%$ teeth & $34.74 \%$ & $15.04 \%$ & \\
\hline CPI 0 & $0 \%$ & $8.9 \%$ & $<0.001$ \\
\hline CPI 1 & $18.6 \%$ & $8.9 \%$ & \\
\hline CPI 2 & $33 \%$ & $23.8 \%$ & \\
\hline CPI 3 & $21.7 \%$ & $52.2 \%$ & \\
\hline CPI 4 & $26.8 \%$ & $6.2 \%$ & \\
\hline Antibody titer [median, Q1-Q3] & $8100(2700-24300)$ & $8100(2700-24300)$ & 0.36 \\
\hline Low $\mathrm{AT} \leq 900$ & 9.3 & 14.2 & 0.13 \\
\hline Moderate AT 2700-8100 & 52.6 & 38.9 & \\
\hline high $A T>8100$ & 38.1 & 46.9 & \\
\hline
\end{tabular}

AT — antibody titter; BOP — bleeding on probing; CAL — clinical attachment loss; CPI — community periodontal index; MI — myocardial infarction; $\mathrm{n}$ - number of participants; $\mathrm{Q}$ - quartile; ${ }^{\mathrm{A}}$ Student $\mathrm{T}$ test

Table 3. Relation between periodontal health parameters, antibody against $P$. gingivalisgingipains and myocardial infarction status.

\begin{tabular}{|c|c|c|c|}
\hline & $\begin{array}{c}\text { Model A } \\
\text { OR }(95 \% \mathrm{Cl})\end{array}$ & $\begin{array}{c}\text { Model B } \\
\text { OR }(95 \% \mathrm{CI})\end{array}$ & $\begin{array}{c}\text { Model C } \\
\text { OR }(95 \% \mathrm{Cl})\end{array}$ \\
\hline Pocket depth $<6 \mathrm{~mm}$ & 1 & 1 & 1 \\
\hline Pocket depth $\geq 6 \mathrm{~mm}$ & $1.98(1.03-3.81)$ & $1.95(0.99-3.82)$ & $1.91(0.95-3.84)$ \\
\hline $\mathrm{CAL} \geq 6$ & 2.35 (1.19-4.67) & $1.31(0.14-1.51)$ & $1.28(1.11-1.49)$ \\
\hline BOP less than $20 \%$ teeth & 1 & 1 & 1 \\
\hline BOP $=20-50 \%$ teeth & $5.26(2.56-10.81)$ & $5.06(2.42-10.58)$ & $4.34(2.00-9.44)$ \\
\hline BOP above $50 \%$ teeth & $5.41(2.60-11.24)$ & $5.02(2.29-11.01)$ & $4.56(2.03-10.27)$ \\
\hline CPI 0 or 1 & 1 & 1 & 1 \\
\hline CPI 2 & $1.32(0.58-3.00)$ & $1.04(0.43-2.47)$ & $0.96(0.39-2.40)$ \\
\hline CPI 3 & $0.40(0.18-0.89)$ & $0.33(0.14-0.79)$ & $0.27(0.11-0.68)$ \\
\hline CPI 4 & 4.13 (1.44-11.87) & $4.03(1.34-12.13)$ & $3.18(1.01-10.06)$ \\
\hline Low $A T \leq 900$ & 1 & 1 & 1 \\
\hline Moderate AT 2700-8100 & $2.06(0.82-5.15)$ & $2.76(1.05-7.24)$ & $2.82(1.02-7.84)$ \\
\hline High AT > 8100 & $1.24(0.49-3.13)$ & $1.48(0.57-3.87)$ & $1.37(0.50-3.76)$ \\
\hline
\end{tabular}

Model A non adjusted

Model B adjusted for: sex, age and years of education

Model C adjusted for: sex, age, years of education, smoking, hypertension, hypercholesterolemia, body mass index, diabetes mellitus and number of teeth

AT — antibody titter; BOP — bleeding on probing; CAL — clinical attachment loss; $\mathrm{Cl}$ — confidence interval; CPI — community periodontal index; $\mathrm{n}-$ number of participants; OR — odds ratio

The relationship between $\mathrm{CP}$ and $\mathrm{MI}$ was first reported by Matilla et al. [1] and later confirmed in the studies which showed 1.2-2.0 times increased odds for MI [23, 24]. In contrast, some studies showed negative relationships between periodontal disease and CHD. Hujoel et al. [25] and Tuominen at al. [26] 
reported that $\mathrm{CP}$ was not associated with $\mathrm{CHD}$ after controlling for CVD risk factors. Depending on the indicator, in our study we showed increased odds of past $\mathrm{MI}$ in $\mathrm{CP}$ patients by $1.2-5.0$ times compared to non $\mathrm{CP}$ patients. The inconsistencies in the strength of the relation may be explained partially at least that various indices have been adopted to assess $\mathrm{CP}$. However, a unified indicator for $\mathrm{CP}$ in population studies is not yet defined. Also, definitions of CHD varied among the studies. Some studies included only patients with history of acute MI, whereas others included patients with stable or unstable angina. It was decided to include only patients with verified definite clinical diagnosis of acute MI.

The evidence for the relationships between $\mathrm{CHD}$ and the level of antibody against $P$. gingivalis is inconsistent. In our study we found that high antibody titer against $P$. gingivalis gingipains is related to an almost 3 times increased chance of MI. This confirms the finding of another other study in which males aged 45 to 75 years who were seropositive for $P$. gingivalis had more frequent CHD than those who were seronegative for $P$. gingivalis [27]. Alternatively, some cross-sectional studies have shown no association between antibodies against $P$. gingivalis and $\mathrm{CHD}$, after adjustment for classic CVD risk factors [28, 29].

The present study has several strengths. The main one is the use of antibody titer to investigate associations between $\mathrm{CP}$ and MI. The use of definition of $\mathrm{CP}$ based on clinical parameters, i.e. pocket depth, to assess relationships between $\mathrm{CP}$ and other chronic diseases has been criticized. Clinical parameters might not be a good indicator of the effect of long-lasting influence of bacterial infection. Thus, antibody titer seems to be a better marker of immunological response for periodontal bacteria. Another strength of this study is that cases and controls were highly comparable. Patients after MI were selected from patients from 5 clinical cardiology wards serving a defined geographical area and the controls were sex, age place of residence matched, and constituted of a random sample of high risk persons registered in selected primary practices serving the same residential area. Restriction of the control group to persons at high risk allowed us to limit confounding by CVD risk factors which was controlled further by adjustment for these in the multivariate analyses.

However case-control design which does not allow for firm conclusions on causality remains the main limitation of the study. Furthermore, the study sample had limited statistical power, although it appeared to be sufficient to find a strong association. Also, the strong relation between $\mathrm{BOP}$ and past MI might be explained at least partially by the use of antiplatelet agents. We were not able to adjust for this this effect as nearly all MI participants were taking these agents. However, the relation between BOP and antiplatelet treatment was not confirmed unequivocally [30]. It is also possible that patients after MI had been paying less attention to oral hygiene as a result of their history of serious disease and as in a consequence had developed more severe $\mathrm{CP}$ than healthy persons. Antibody levels for periodontopatic bacteria are considered to be stable over time, but it remained unclear whether antibody level is an indicator of disease history or active infection. The other limitation is that the present study assessed only antibody for one periodontopathogenic bacteria. It is possible that if the presence of antibodies against other periodontitis-associated bacterial species were included in the analysis the relationships could have been stronger.

\section{Conclusions}

In conclusion, an association was found between chronic periodontal disease and past MI which was independent of classical CVD risk factors and confirmed by the association between past MI and immunological reactions against $P$. gingivalis gingipains.

\section{Acknowledgements}

Authors express special thanks to all participants of the study.

This publication was supported by the Faculty of Medicine, Jagiellonian University Medical College, Leading National Research Centre (KNOW) 2012-2017.

Funding: This study was supported by the Polish National Science Centre decision no: DEC 2011/03/B/NZ7/06101 and a grant of: Jagiellonian University Medical College project no: K/ZDS/004092.

Conflict of interest: Andrzej Pająk declared honorarium for consultancy from AMGEN not related with submitted work. The other authors declare no conflict of interest. 


\section{References}

1. Mattila KJ, Nieminen MS, Valtonen VV, et al. Association between dental health and acute myocardial infarction. BMJ. 1989; 298(6676): 779-781, doi: 10.1136/bmj.298.6676.779, indexed in Pubmed: 2496855.

2. Humphrey LL, Fu R, Buckley DI, et al. Periodontal disease and coronary heart disease incidence: a systematic review and meta-analysis. J Gen Intern Med. 2008; 23(12): 2079-2086, doi: 10.1007/s11606-008-0787-6, indexed in Pubmed: 18807098.

3. Teeuw WJ, Slot DE, Susanto H, et al. Treatment of periodontitis improves the atherosclerotic profile: a systematic review and meta-analysis. J Clin Periodontol. 2014; 41(1): 70-79, doi: 10.1111/jcpe.12171, indexed in Pubmed: 24111886.

4. Cotti E, Dessì C, Piras A, et al. Can a chronic dental infection be considered a cause of cardiovascular disease? A review of the literature. Int J Cardiol. 2011; 148(1): 4-10, doi: 10.1016/j. ijcard.2010.08.011, indexed in Pubmed: 20851474.

5. König J, Holtfreter B, Kocher T. Periodontal health in Europe: future trends based on treatment needs and the provision of periodontal services-position paper 1. Eur J Dent Educ. 2010; 14 Suppl 1: 4-24, doi: 10.1111/j.1600-0579.2010.00620.x, indexed in Pubmed: 20415972.

6. http://www.mz.gov. pl/zdrowie-i-profilaktyka/programy-zdrowotne/wykaz-programow/monitorowanie-stanu-zdrowia-jamyustnej-populacji-polskiej-w-latach-2013-2015, entered: 10; 08: 2015.

7. Holt SC, Ebersole JL. Porphyromonas gingivalis, Treponema denticola, and Tannerella forsythia: the "red complex", a prototype polybacterial pathogenic consortium in periodontitis. Periodontol. 2000. 2005; 38: 72-122, doi: 10.1111/j.16000757.2005.00113.x, indexed in Pubmed: 15853938.

8. Yang HW, Huang YF, Chou MY. Occurrence of Porphyromonas gingivalis and Tannerella forsythensis in periodontally diseased and healthy subjects. J Periodontol. 2004; 75(8): 1077-1083, doi: 10.1902/jop.2004.75.8.1077, indexed in Pubmed: 15455734 .

9. Lamont RJ, Jenkinson HF. Life below the gum line: pathogenic mechanisms of Porphyromonas gingivalis. Microbiol Mol Biol Rev. 1998; 62(4): 1244-1263, doi: 10.3389/fmicb.2016.00053, indexed in Pubmed: 9841671.

10. Potempa J, Pike R, Travis J. Titration and mapping of the active site of cysteine proteinases from Porphyromonas gingivalis (gingipains) using peptidyl chloromethanes. Biol Chem. 1997; 378(3-4): 223-230, doi: 10.1515/bchm.1997.378.3-4.223, indexed in Pubmed: 9165075.

11. Friedewald VE, Kornman KS, Beck JD, et al. American Journal of Cardiology, Journal of Periodontology. The American Journal of Cardiology and Journal of Periodontology Editors' Consensus: periodontitis and atherosclerotic cardiovascular disease. Am J Cardiol. 2009; 104(1): 59-68, doi: 10.1016/j.amjcard.2009.05.002, indexed in Pubmed: 19576322.

12. Tam V, O’Brien-Simpson NM, Chen YY, et al. The RgpA-Kgp proteinase-adhesin complexes of Porphyromonas gingivalis Inactivate the Th2 cytokines interleukin-4 and interleukin-5. Infect Immun. 2009; 77(4): 1451-1458, doi: 10.1128/IAI.01377-08, indexed in Pubmed: 19168731.

13. Fagundes JA, Monoo LD, Euzébio Alves VT, et al. Porphyromonas gingivalis is associated with protease-activated receptor-2 upregulation in chronic periodontitis. J Periodontol. 2011; 82(11): 1596-1601, doi: 10.1902/jop.2011.110073, indexed in Pubmed: 21513479.

14. Higashi Y, Goto C, Jitsuiki D, et al. Periodontal infection is associated with endothelial dysfunction in healthy subjects and hypertensive patients. Hypertension. 2008; 51(2): 446-453, doi: 10.1161/HYPERTENSIONAHA.107.101535, indexed in Pubmed: 18039979.

15. Haraszthy VI, Zambon JJ, Trevisan M, et al. Identification of periodontal pathogens in atheromatous plaques. J Periodontol. 2000; 71(10): 1554-1560, doi: 10.1902/jop.2000.71.10.1554, indexed in Pubmed: 11063387.

16. Brodala N, Merricks EP, Bellinger DA, et al. Porphyromonas gingivalis bacteremia induces coronary and aortic atherosclerosis in normocholesterolemic and hypercholesterolemic pigs. Arterioscler Thromb Vasc Biol. 2005; 25(7): 1446-1451, doi: 10.1161/01. ATV.0000167525.69400.9c, indexed in Pubmed: 15845905.

17. Craig RG, Boylan R, Yip J, et al. Serum IgG antibody response to periodontal pathogens in minority populations: relationship to periodontal disease status and progression. J Periodont Res. 2002; 37(2): 132-146, doi: 10.1034/j.1600-0765.2002.00031.x, indexed in Pubmed: 12009183.

18. Lwanga SK, Lemeshow S. Sample size determination in health studies. World Health Organization, Geneva, 1991.

19. Organization W. Oral Health Surveys: Basic Methods. Biometrics. 1971; 27(4): 1111, doi: 10.2307/2528861.

20. Ainamo J, Bay I. Problems and proposals for recording gingivitis and plaque. Int Dent J. 1975; 25(4): 229-235, indexed in Pubmed: 1058834 .

21. Ainamo J, Barmes D, Beagrie G, et al. Development of the World Health Organization (WHO) community periodontal index of treatment needs (CPITN). Int Dent J. 1982; 32(3): 281-291, doi: 10.1111/j.1600-0528.1986.tb01492.x, indexed in Pubmed: 6958657.

22. Zdzalik M, Karim AY, Wolski K, et al. Prevalence of genes encoding extracellular proteases in Staphylococcus aureus - important targets triggering immune response in vivo. FEMS Immunol Med Microbiol. 2012; 66(2): 220-229, doi: 10.1111/j.1574695X.2012.01005.x, indexed in Pubmed: 22762789.

23. Briggs JE, McKeown PP, Crawford VLS, et al. Angiographically confirmed coronary heart disease and periodontal disease in middle-aged males. J Periodontol. 2006; 77(1): 95-102, doi: 10.1902/ jop.2006.77.1.95, indexed in Pubmed: 16579709.

24. Oe Y, Soejima H, Nakayama H, et al. Significant association between score of periodontal disease and coronary artery disease. Heart Vessels. 2009; 24(2): 103-107, doi: 10.1007/s00380-0081096-z, indexed in Pubmed: 19337793.

25. Hujoel PP, Drangsholt M, Spiekerman C, et al. Periodontal disease and coronary heart disease risk. JAMA. 2000; 284(11): 1406-1410, doi: 10.1001/jama.284.11.1406, indexed in Pubmed: 10989403.

26. Tuominen R, Reunanen A, Paunio M, et al. Oral health indicators poorly predict coronary heart disease deaths. J Dent Res. 2003 82(9): 713-718, doi: 10.1177/154405910308200911, indexed in Pubmed: 12939356.

27. Pussinen PJ, Jousilahti P, Alfthan G, et al. Antibodies to periodontal pathogens are associated with coronary heart disease. Arterioscler Thromb Vasc Biol. 2003; 23(7): 1250-1254, doi: 10.1161/01. ATV.0000072969.71452.87, indexed in Pubmed: 12714435.

28. Beck JD, Eke P, Heiss G, et al. Periodontal disease and coronary heart disease: a reappraisal of the exposure. Circulation. 2005, 112(1): 19-24, doi: 10.1161/CIRCULATIONAHA.104.511998, indexed in Pubmed: 15983248 .

29. Holmlund A, Hedin M, Pussinen PJ, et al. Porphyromonas gingivalis $(\mathrm{Pg})$ a possible link between impaired oral health and acute myocardial infarction. Int J Cardiol. 2011; 148(2): 148-153, doi: 10.1016/j.ijcard.2009.10.034, indexed in Pubmed: 19913930.

30. Schrodi J, Recio L, Fiorellini J, et al. The effect of aspirin on the periodontal parameter bleeding on probing. J Periodontol. 2002; 73(8): 871-876, doi: 10.1902/jop.2002.73.8.871, indexed in Pubmed: 12211496. 